\title{
ALGEBRAS ON THE DISK AND DOUBLY COMMUTING MULTIPLICATION OPERATORS
}

\author{
SHELDON AXLER AND PAMELA GORKIN
}

\begin{abstract}
We prove that a bounded analytic function $f$ on the unit disk is in the little Bloch space if and only if the uniformly closed algebra on the disk generated by $H^{\infty}$ and $\bar{f}$ does not contain the complex conjugate of any interpolating Blaschke product. A version of this result is then used to prove that if $f$ and $g$ are bounded analytic functions on the unit disk such that the commutator $T_{f} T_{g}^{*}-T_{g}^{*} T_{f}$ (here $T_{f}$ denotes the operator of multiplication by $f$ on the Bergman space of the disk) is compact, then $\left(1-|z|^{2}\right) \min \left\{\left|f^{\prime}(z)\right|,\left|g^{\prime}(z)\right|\right\} \rightarrow 0$ as $|z| \uparrow 1$.
\end{abstract}

1. Introduction. Let $D$ denote the open unit disk in the complex plane, and let $d A$ denote the usual two-dimensional Lebesgue area measure on $D$. The algebra of bounded analytic functions on the disk $D$ is called $H^{\infty}$. For now, we think of $H^{\infty}$ as a closed subalgebra of $L^{\infty}(D, d A)$. If $u \in L^{\infty}(D, d A)$, then the closed subalgebra of $L^{\infty}(D, d A)$ generated by $H^{\infty}$ and $u$ is denoted by $H^{\infty}[u]$.

The little Bloch space is the set of analytic functions $f$ on the disk $D$ such that

$$
\left(1-|z|^{2}\right) f^{\prime}(z) \rightarrow 0 \quad \text { as }|z| \uparrow 1 .
$$

An interpolating sequence is a sequence $\left\{w_{n}\right\}_{n=1}^{\infty}$ in $D$ such that for every bounded sequence $\left\{c_{n}\right\}_{n=1}^{\infty}$ of complex numbers, there is a function $f \in H^{\infty}$ such that $f\left(w_{n}\right)=c_{n}$ for every positive integer $n$. In this paper, a finite sequence in $D$ is not considered to be an interpolating sequence. An interpolating Blaschke product $b$ is a function on $D$ of the form

$$
b(z)=\prod_{n=1}^{\infty} \frac{\left|w_{n}\right|}{w_{n}} \frac{w_{n}-z}{1-\overline{w_{n}} z},
$$

where $\left\{w_{n}\right\}_{n=1}^{\infty}$ is an interpolating sequence (and $0 / 0$ is defined to equal 1). Carleson [6] proved that a sequence $\left\{w_{n}\right\}_{n=1}^{\infty}$ in $D$ is interpolating if and only if

$$
\inf _{n} \prod_{\substack{m=1 \\ m \neq n}}^{\infty}\left|\frac{w_{m}-w_{n}}{1-\overline{w_{m}} w_{n}}\right|>0 .
$$

An infinite sequence $\left\{w_{n}\right\}_{n=1}^{\infty}$ in $D$ is called thin if

$$
\prod_{\substack{m=1 \\ m \neq n}}^{\infty}\left|\frac{w_{m}-w_{n}}{1-\overline{w_{m}} w_{n}}\right| \rightarrow 1 \quad \text { as } n \rightarrow \infty .
$$

Received by the editors August 7, 1987.

1980 Mathematics Subject Classification (1985 Revision). Primary 46J15, 47B35, 30D50.

The first author was supported in part by the National Science Foundation. 
Clearly every thin sequence is interpolating. The Blaschke product associated with a thin sequence is called a thin Blaschke product.

In this paper we prove that if $f \in H^{\infty}$ and $\left\{w_{n}\right\}_{n=1}^{\infty}$ is a thin sequence such that

$$
\liminf _{n \rightarrow \infty}\left(1-\left|w_{n}\right|^{2}\right)\left|f^{\prime}\left(w_{n}\right)\right|>0
$$

then the complex conjugate of the Blaschke product associated with $\left\{w_{n}\right\}_{n=1}^{\infty}$ is contained in $H^{\infty}[\bar{f}]$. This will allow us to prove, among other results, that if $f \in H^{\infty}$, then $H^{\infty}[\bar{f}]$ contains the complex conjugate of an interpolating Blaschke product if and only if $f$ is not in the little Bloch space.

Our results were motivated by questions about multiplication operators on Bergman spaces. The Bergman space $L_{a}^{2}$ is the set of analytic functions $f$ on $D$ such that $f \in L^{2}(D, d A)$. The Bergman space is a closed subspace of $L^{2}(D, d A)$, and so $L_{a}^{2}$ is a Hilbert space. For $f \in H^{\infty}$, the multiplication operator $T_{f}$ from $L_{a}^{2}$ to $L_{a}^{2}$ is defined by $T_{f} h=f h$.

We prove that if $f$ and $g$ are $H^{\infty}$ functions such that $T_{f} T_{g}^{*}-T_{g}^{*} T_{f}$ is compact, then

$$
\left(1-|z|^{2}\right) \min \left\{\left|f^{\prime}(z)\right|,\left|g^{\prime}(z)\right|\right\} \rightarrow 0 \quad \text { as }|z| \uparrow 1 .
$$

The next section presents the machinery we need. $\S 3$ contains a statement of the main results along with a discussion about what we have actually proved. $\S 4$ contains proofs of the results that deal only with algebras on the disk. $\S 5$ contains proofs of the results concerning multiplication operators. The paper concludes with a section of questions and comments.

2. The maximal ideal space of $H^{\infty}$ and its parts. We will need to work on the maximal ideal space of $H^{\infty}$, which is denoted by $M$ and is defined to be the set of multiplicative linear maps from $H^{\infty}$ onto the field of complex numbers. Each multiplicative linear functional $\varphi \in M$ has norm 1 (as an element of the dual of $H^{\infty}$ ). If we think of $M$ as a subset of the dual of $H^{\infty}$ with the weak-star topology, then $M$ becomes a compact Hausdorff space. Explicitly, a net $\left\{\varphi_{\alpha}\right\}$ in $M$ (we usually do not display the index set of a net) converges to $\varphi$ in $M$ if and only if

$$
\varphi_{\alpha}(f) \rightarrow \varphi(f) \text { for every } f \in H^{\infty} .
$$

If $w$ is a point in the unit disk $D$, then point evaluation at $w$ is a multiplicative linear functional on $H^{\infty}$, and so we can think of $w$ as an element of $M$. Thus we will freely think of the disk $D$ as a subset of the maximal ideal space $M$. The topology that $D$ inherits as a subset of $M$ coincides with the usual topology on $D$. Without further comment, we will often use Lennart Carleson's Corona Theorem [7], which states that the disk $D$ is a dense subset of the maximal ideal space $M$.

By using the Gelfand transform, we can think of $H^{\infty}$ as a subset of $C(M)$, the continuous, complex-valued functions on the maximal ideal space of $H^{\infty}$. Explicitly, for $f \in H^{\infty}$, we extend $f$ from $D$ to $M$ by defining

$$
f(\varphi)=\varphi(f) \text { for every } \varphi \in M .
$$

Note that this definition is consistent with our earlier identification of $D$ with a subset of $M$. We will use the notations $f(\varphi)$ and $\varphi(f)$ interchangeably; $f(\varphi)$ will be used when we need to think of $f$ as a function on $M$, and $\varphi(f)$ will be used when we want to think of $\varphi$ as a function on $H^{\infty}$. 
If $u$ is a bounded, complex-valued harmonic function on $D$, then $u$ extends to be a continuous, complex-valued function on $M[13$, Lemma 4.4]. Thus we can think of $H^{\infty}[u]$ as a closed subalgebra of $C(M)$.

For $\varphi, \tau \in M$, the pseudohyperbolic distance between $\varphi$ and $\tau$, denoted $\rho(\varphi, \tau)$ is defined by

$$
\rho(\varphi, \tau)=\sup \left\{|\varphi(f)|: f \in H^{\infty},|f|<1 \text { on } D, \text { and } \tau(f)=0\right\} .
$$

If $w, z \in D \subset M$, then the Schwarz Lemma can be used to show that

$$
\rho(w, z)=\left|\frac{w-z}{1-\bar{w} z}\right| .
$$

Although $\rho$ is a metric on $M[\mathbf{1 1}$, p. 401], whenever we refer to any topological property on $M$ (convergence, continuity, etc.), we mean the usual topology on $M$ introduced earlier (the weak-star topology inherited from the dual of $H^{\infty}$ ). The usual topology on $M$ is not the same as the topology induced by the metric $\rho$. In fact, $M$ is not metrizable in its usual topology, which is why we often need to use nets rather than sequences.

For $\varphi \in M$, the part of $\varphi$ (sometimes called the Gleason part of $\varphi$ ) is denoted by $P(\varphi)$ and is defined by

$$
P(\varphi)=\{\tau \in M: \rho(\varphi, \tau)<1\} .
$$

Any two parts are either disjoint or equal [11, pp. 401-402]. If $w \in D \subset M$, then $P(w)=D$.

For each $\varphi \in M$, Kenneth Hoffman [13] constructed a canonical map $L_{\varphi}$ of the disk $D$ onto the part $P(\varphi)$. This map is defined by taking a net $\left\{w_{\alpha}\right\}$ in $D$ such that $w_{\alpha} \rightarrow \varphi$, and defining

$$
\left(L_{\varphi}(z)\right)(f)=\lim _{\alpha} f\left(\frac{w_{\alpha}+z}{1+\overline{w_{\alpha}} z}\right)
$$

for $z \in D$ and $f \in H^{\infty}$. The above limit exists and is independent of the net $\left\{w_{\alpha}\right\}$, provided that $w_{\alpha} \rightarrow \varphi$. For each $f \in H^{\infty}$, the composition $f \circ L_{\varphi}$ is in $H^{\infty}$. In addition to proving that $L_{\varphi}$ maps $D$ onto the part $P(\varphi)$, Hoffman [13] proved that $L_{\varphi}$ has many remarkable properties. For example, either $L_{\varphi}$ is a constant map or $L_{\varphi}$ is injective; in the latter case $P(\varphi)$ is called an analytic disk. We will need to use Hoffman's main result, which states that $P(\varphi)$ is an analytic disk if and only if there is an interpolating sequence containing $\varphi$ in its closure. In addition to Hoffman's paper, a nice exposition of these results can be found in [11, Chapter $\mathrm{X}]$.

3. Main results. Theorems 1,2 , and 3 contain the main results in this paper. We emphasize that not all the equivalences in Theorems 2 and 3 are due to us. We have included results due to others in the statements of Theorems 2 and 3 so that the reader can view the overall context. Proper attribution of the various equivalences of Theorems 2 and 3 is contained in the discussion following the statements of the theorems.

THEOREM 1. Let $f \in H^{\infty}$. Let $\left\{w_{n}\right\}_{n=1}^{\infty}$ be a thin sequence in $D$ and let $b$ be the associated Blaschke product. If

$$
\liminf _{n \rightarrow \infty}\left(1-\left|w_{n}\right|^{2}\right)\left|f^{\prime}\left(w_{n}\right)\right|>0,
$$

then the complex conjugate of $b$ is contained in $H^{\infty}[\bar{f}]$. 
THEOREM 2. Let $f \in H^{\infty}$. The following are equivalent:

(2.a) $\left(1-|z|^{2}\right) f^{\prime}(z) \rightarrow 0$ as $|z| \uparrow 1$;

(2.b) for each $\varphi \in M \backslash D, f$ is constant on $P(\varphi)$;

(2.c) $H^{\infty}[\bar{f}]$ is contained in $\left\{u \in C(M): u \circ L_{\varphi} \in H^{\infty}\right.$ for every $\left.\varphi \in M \backslash D\right\}$;

(2.d) $\left\{h \in H^{\infty}: \bar{h} \in H^{\infty}[\bar{f}]\right\}$ is contained in the little Bloch space;

(2.e) $H^{\infty}[\bar{f}]$ does not contain the complex conjugate of any interpolating Blaschke product;

(2.f) $H^{\infty}[\bar{f}]$ does not contain the complex conjugate of any thin Blaschke product.

(2.g) $T_{f} T_{f}^{*}-T_{f}^{*} T_{f}$ is compact.

THEOREM 3. Let $f, g \in H^{\infty}$. The following are equivalent:

(3.a) $\left(1-|z|^{2}\right) \min \left\{\left|f^{\prime}(z)\right|,\left|g^{\prime}(z)\right|\right\} \rightarrow 0$ as $|z| \uparrow 1$;

(3.b) for each $\varphi \in M \backslash D$, either $f$ or $g$ is constant on $P(\varphi)$;

(3.c) $H^{\infty}[\bar{f}] \cap H^{\infty}[\bar{g}]$ is contained in $\left\{u \in C(M): u \circ L_{\varphi} \in H^{\infty}\right.$ for every $\varphi \in M \backslash D\}$

(3.d) $\left\{h \in H^{\infty}: \bar{h} \in H^{\infty}[\bar{f}] \cap H^{\infty}[\bar{g}]\right\}$ is contained in the little Bloch space;

(3.e) $H^{\infty}[\bar{f}] \cap H^{\infty}[\bar{g}]$ does not contain the complex conjugate of any interpolating Blaschke product;

(3.f) $H^{\infty}[\bar{f}] \cap H^{\infty}[\bar{g}]$ does not contain the complex conjugate of any thin Blaschke product;

(3.g) $T_{f} T_{g}^{*}-T_{g}^{*} T_{f}$ is compact.

Of course Theorem 2 follows immediately from Theorem 3 by letting $g$ equal $f$ in Theorem 3 . We discovered Theorem 1 while attempting to prove the implication (3.g) $\Rightarrow(3 . a)$. The equivalence (3.g) $\Leftrightarrow(3 . a)$ does not easily follow from the equivalence (2.g) $\Leftrightarrow(2 . \mathrm{a})$ (or its proof). However, the statement that (3.a) through (3.f) are all equivalent to each other is not harder to prove than the statement that (2.a) through (2.f) are all equivalent to each other.

The equivalence of (2.g) to (2.a) is due to Axler [1, Theorem 7]. Clearly (2.a) is simply the statement that $f$ is in the little Bloch space. Many other conditions are known to be equivalent to (2.a); for example see [1, Theorem 2] and [19]. The equivalence of (2.a) to (2.b) has long been known.

The equivalence of (2.a) to (2.c)-(2.f) is new and will be proved later in this paper by showing that (3.a) is equivalent to (3.c)-(3.f). We believe that the equivalence of conditions (2.a) through (2.f) suggests that closed subalgebras of $L^{\infty}(D, d A)$ generated by $H^{\infty}$ and conjugate analytic functions might have a rich structure comparable to the structure of the boundary-value algebras described by the ChangMarshall Theorem; these matters are discussed in more detail in the last section of the paper.

The equivalence of (3.g) to (3.a) was conjectured by Axler [1, §4]. Before dealing with (3.g), we will prove that (3.a) through (3.f) are all equivalent to each other. Then we will prove the implication (3.g) $\Rightarrow(3 . \mathrm{a})$; this result was also obtained independently at the same time by Dechao Zheng [22]. Zheng's proof uses techniques very different from ours. Each proof provides a different insight into the problem. Zheng's techniques allow him to prove that $(3 . \mathrm{a}) \Rightarrow(3 . \mathrm{g})$. Our techniques do not lead to a proof that (3.a) $\Rightarrow(3 . \mathrm{g})$, but Zheng's techniques do not lead to a proof of the equivalence of (3.e) [or (3.f)] to the other conditions of Theorem 3. Partial 
results concerning the compactness of the commutator of a multiplication operator and the adjoint of another multiplication operator on the Bergman space of the unit ball of $\mathbf{C}^{n}$ were proved independently at about the same time by Keiji Izuchi in $[\mathbf{1 4}, \S 5]$.

4. Algebras on the disk. In this section we will prove Theorem 1 and also prove that (3.a) through (3.f) of Theorem 3 are all equivalent to each other.

For $\varphi \in M \backslash D$ and $f \in H^{\infty}$, we will need to know a formula for the derivative of $f \circ L_{\varphi}$. Let $\left\{w_{\alpha}\right\}$ be a net in $D$ such that $w_{\alpha} \rightarrow \varphi$ in $M$ (which implies that $\left.\left|w_{\alpha}\right| \rightarrow 1\right)$. For $w \in D$, recall that $\left(f \circ L_{\varphi}\right)(w)$ was defined by

$$
\left(f \circ L_{\varphi}\right)(w)=\lim _{\alpha} f\left(\frac{w_{\alpha}+w}{1+\overline{w_{\alpha}} w}\right) .
$$

Differentiate both sides of the above equation with respect to $w$, obtaining

$$
\left(f \circ L_{\varphi}\right)^{\prime}(w)=\lim _{\alpha} \frac{1-\left|w_{\alpha}\right|^{2}}{\left(1+\overline{w_{\alpha}} w\right)^{2}} f^{\prime}\left(\frac{w_{\alpha}+w}{1+\overline{w_{\alpha}} w}\right) .
$$

Letting

$$
z_{\alpha}=\frac{w_{\alpha}+w}{1+\overline{w_{\alpha}} w}
$$

(note that $\left|z_{\alpha}\right| \rightarrow 1$ ), the above formula for $\left(f \circ L_{\varphi}\right)^{\prime}(w)$ becomes

$$
\left|\left(f \circ L_{\varphi}\right)^{\prime}(w)\right|=\lim _{\alpha} \frac{\left(1-\left|z_{\alpha}\right|^{2}\right)\left|f^{\prime}\left(z_{\alpha}\right)\right|}{1-|w|^{2}} .
$$

We need one lemma before beginning the proof of Theorem 1. If $f \in H^{\infty}$, then we can think of $H^{\infty}[\bar{f}]$ as a closed subalgebra of $C(M)$, and thus $M$ is contained in the maximal ideal space of $H^{\infty}[\bar{f}]$. Conversely, if $\varphi$ is a multiplicative linear functional on $H^{\infty}[\bar{f}]$, then clearly $\varphi \mid H^{\infty}$ is a multiplicative linear functional on $H^{\infty}$. If no two distinct multiplicative linear functionals on $H^{\infty}[\bar{f}]$ restrict the same multiplicative linear functional on $H^{\infty}$, then we will have an identification of the maximal ideal space of $H^{\infty}[\bar{f}]$ with $M$. The following lemma shows that this is the case.

LemMa 4. Let $f \in H^{\infty}$. Then the maximal ideal space of $H^{\infty}[\bar{f}]$ equals $M$. More precisely, each multiplicative linear functional on $H^{\infty}$ has a unique extension to a multiplicative linear functional on $H^{\infty}[\bar{f}]$, and every multiplicative linear functional on $H^{\infty}[\bar{f}]$ is obtained in this fashion.

ProOF. Let $\varphi$ be a multiplicative linear functional on $H^{\infty}[\bar{f}]$. As discussed above, we need only prove that $\varphi$ is uniquely determined by $\varphi \mid H^{\infty}$. Using the usual description of the dual of $C(M)$, we see that $\varphi$ (as a linear functional on $\left.H^{\infty}[\bar{f}]\right)$ can be represented by integration against a positive Borel measure on $M$, and so $\varphi(\bar{f})=\overline{\varphi(f)}$. Because $f \in H^{\infty}$, this means that $\varphi(\bar{f})$ is uniquely determined by $\varphi \mid H^{\infty}$, completing the proof.

For $f \in H^{\infty}$ and $\varphi \in M$, the order of the zero of $f$ at $\varphi$, denoted $\operatorname{ord}(f ; \varphi)$, is defined to be the supremum of the positive integers $n$ such that $f$ can be factored as $f=f_{1} f_{2} \cdots f_{n}$, where $\varphi\left(f_{k}\right)=0$ for $k=1, \ldots, n$; if $\varphi(f) \neq 0$, then we interpret the above definition to mean that $\operatorname{ord}(f ; \varphi)=0$. Hoffman's paper [13], which we heavily use, works with a slightly different definition of the order of a zero (see [13, 
Lemma 2.3], for Hoffman's definition). We have chosen a slightly simpler definition. The results in [13], particularly Theorem 5.4 and the comment following its proof, show that the two definitions are equivalent.

We are now ready to prove Theorem 1 , which we restate here for convenience.

THEOREM 1. Let $f \in H^{\infty}$. Let $\left\{w_{n}\right\}_{n=1}^{\infty}$ be a thin sequence in $D$ and let $b$ be the associated Blaschke product. If

$$
\liminf _{n \rightarrow \infty}\left(1-\left|w_{n}\right|^{2}\right)\left|f^{\prime}\left(w_{n}\right)\right|>0,
$$

then the complex conjugate of $b$ is contained in $H^{\infty}[\bar{f}]$.

PROOF. Let

$$
E=\{\varphi \in M: f \text { is not constant on } P(\varphi)\} .
$$

If $\varphi \in M \backslash D$ and $\varphi(b)=0$, then $\varphi$ is in the closure (in $M$ ) of $\left\{w_{n}\right\}_{n=1}^{\infty}[\mathbf{1 1}$, Chapter IX, Lemma 3.3]; our formula for the derivative of $f \circ L_{\varphi}$ and the condition $\liminf _{n \rightarrow \infty}\left(1-\left|w_{n}\right|^{2}\right)\left|f^{\prime}\left(w_{n}\right)\right|>0$ imply that $\left(f \circ L_{\varphi}\right)^{\prime}(0) \neq 0$, so $f$ is not constant on $P(\varphi)$. Thus all the zeroes of $b$ (in $M$ ) are contained in $E$.

Let $S$ be a subset of $M$ that is a maximal antisymmetric set for $H^{\infty}[\bar{f}]$; this means that every function in $H^{\infty}[\bar{f}]$ that is real valued on $S$ is constant on $S$ and that no subset of $M$ properly containing $S$ has this property. By Bishop's Antisymmetric Decomposition Theorem [10, Theorem 13.1], to conclude that $\bar{b} \in$ $H^{\infty}[\bar{f}]$ we need only show that $\bar{b}\left|S \in H^{\infty}[\bar{f}]\right| S$.

First suppose that $S \cap E$ is the empty set. Because $E$ contains all the zeroes of $b$ (in $M$ ), $b$ cannot vanish on $S$. Because $S$ is a weak-peak set for the algebra $H^{\infty}[\bar{f}]$ [10, Theorem 13.1], Lemma 4 implies that the maximal ideal space of the Banach algebra $H^{\infty}[\bar{f}] \mid S$ equals $S$. Thus $b \mid S$ does not vanish on the maximal ideal space of $H^{\infty}[\bar{f}] \mid S$, and so $(1 / b)\left|S \in H^{\infty}[\bar{f}]\right| S$.

If $\varphi \in S$, then (because $E$ consists of a union of parts) $P(\varphi) \cap E$ is the empty set. If a thin Blaschke product does not vanish anywhere on a part, then it must have modulus one everywhere on that part (see [5, Proposition 3.9] or [12, Proposition 2.3]). Thus

$$
\bar{b}|S=(1 / b)| S \in H^{\infty}[\bar{f}] \mid S,
$$

completing the proof in the case where $S \cap E$ is the empty set.

Now suppose that $S \cap E$ is nonempty. Because $S$ is an antisymmetric set for $H^{\infty}[\bar{f}]$, there is a complex constant $c$ such that $f \mid S=c$. Let

$$
N=\min \{\operatorname{ord}(f-c ; \varphi): \varphi \in S\} .
$$

By [13, Lemma 2.3], ord $(f-c ; \varphi)$ is finite for every $\varphi$ in $E$. Thus $N<\infty$.

Let $\varphi \in S$ be such that ord $(f-c ; \varphi)=N$. Let $b_{0}$ denote the Blaschke factor of $f-c$, so $(f-c) / b_{0}$ has no zeroes on $D$. By [13, Lemma 2.2], $\varphi\left((f-c) / b_{0}\right) \neq 0$. From the equation $f-c=b_{0}\left((f-c) / b_{0}\right)$, we can conclude that $\operatorname{ord}\left(b_{0}, \varphi\right)=N$. Repeated applications of $\left[\mathbf{1 3}\right.$, Theorem 5.3] to $b_{0}$ now show that there are interpolating Blaschke products $b_{1}, \ldots, b_{N}$ and a function $h \in H^{\infty}$ such that $\varphi\left(b_{j}\right)=0$ for $j=1, \ldots, n$ and $f-c=b_{1} \cdots b_{N} h$. The function $b_{N} h$ is identically 0 on $S$, because otherwise $f-c$ would have a zero on $S$ of order less than $N$, contradicting the definition of $N$. 
For the multiplicative linear functional $\varphi$ constructed in the above paragraph, we have $\varphi \in\left\{\beta \in M: \operatorname{ord}\left(b_{N} h ; \beta\right)<2\right\}$. Let

$$
g(z)=\left(1-|z|^{2}\right)\left(b_{N} h\right)^{\prime}(z) .
$$

Clearly $g$ is a continuous function on $D$. By [13, Theorem 4.5], $g$ extends to a continuous complex-valued function on $M$. The set $\left\{\beta \in M: \operatorname{ord}\left(b_{N} h ; \beta\right)<2\right\}$ is open in $M$, because by [13, Theorem 5.4] it is equal to $\left\{\beta \in M:\left(b_{N} h\right)(\beta) \neq\right.$ $0\} \cup\{\beta \in M: g(\beta) \neq 0\}$.

Because $b_{N}$ is an interpolating Blaschke product and because $\varphi\left(b_{N}\right)=0, \varphi$ is in the closure (in $M$ ) of the zeroes of $b_{N}$. Thus there is a sequence $\left\{z_{n}\right\}_{n=1}^{\infty}$ in $D$ such that each $z_{n}$ is a zero of $b_{N}$ and

$$
\varphi \in \overline{\left\{z_{n}\right\}_{n=1}^{\infty}} \subset\left\{\beta \in M: \operatorname{ord}\left(b_{N} h ; \beta\right)<2\right\} ;
$$

here $\overline{\left\{z_{n}\right\}_{n=1}^{\infty}}$ denotes the closure of $\left\{z_{n}\right\}_{n=1}^{\infty}$ in $M$. Let $p$ be the interpolating Blaschke product whose zeroes are $\left\{z_{n}\right\}_{n=1}^{\infty}$, and let $q \in H^{\infty}$ be such that $b_{N} h=p q$. Thus

$$
S=\{\tau \in S: \tau(p)=0\} \cup\{\tau \in S: \tau(q)=0\} .
$$

If $\tau \in M$ and $\tau(p)=0$, then (because $p$ is an interpolating Blaschke product)

$$
\tau \in \overline{\left\{z_{n}\right\}_{n=1}^{\infty}} \subset\left\{\beta \in M: \operatorname{ord}\left(b_{N} h ; \beta\right)<2\right\}
$$

and thus $\tau(q) \neq 0$. Thus $\{\tau \in S: \tau(p)=0\}$ and $\{\tau \in S: \tau(q)=0\}$ are disjoint closed sets.

As noted earlier in this proof, the maximal ideal space of $H^{\infty}[\bar{f}] \mid S$ is $S$. If $S$ were disconnected, then by the Shilov Idempotent Theorem [10, Corollary 6.5], $H^{\infty}[\bar{f}] \mid S$ would contain a nontrivial characteristic function, which would contradict the antisymmetry of $S$. Thus $S$ is connected, so one of the sets $\{\tau \in S: \tau(p)=0\}$ and $\{\tau \in S: \tau(q)=0\}$ is $S$ and the other is empty. Because $\varphi$ is in the first set, $\{\tau \in S: \tau(p)=0\}$ must equal $S$, so $S \subset\{\tau \in M: \tau(p)=0\}$. that

Because $\left\{z_{n}\right\}_{n=1}^{\infty}$ is an interpolating sequence, there is a function $g \in H^{\infty}$ such

$$
g\left(z_{n}\right)=\bar{b}\left(z_{n}\right) \quad \text { for every positive integer } n .
$$

Because $S$ is contained in the closure (in $M$ ) of $\left\{z_{n}\right\}_{n=1}^{\infty}$, we see that $\bar{b}|S=g| S \in$ $H^{\infty} \mid S$, completing the proof.

The following corollary of Theorem 1 asserts that the complex conjugate of every thin subproduct of an interpolating Blaschke product $b$ is in the algebra generated by $H^{\infty}$ and $\bar{b}$. This result need not be true if the Blaschke product $b$ is not interpolating. For example, let $b$ be a Blaschke product (with infinitely many zeroes) in the little Bloch space (such Blaschke products exist; see [18]). The implication (2.a) $\Rightarrow(2 . \mathrm{f})$ of Theorem 2 shows that the complex conjugate of no thin subproduct of $b$ is in $H^{\infty}[\bar{b}]$.

COROLlARY 5. Let $b$ be an interpolating Blaschke product. Then the complex conjugate of every thin subproduct of $b$ is in $H^{\infty}[\bar{b}]$.

PROOF. Let the interpolating Blaschke product $b$ of Corollary 5 play the role of $f$ in Theorem 1. Carleson's interpolating condition implies that the hypothesis of Theorem 1 is satisfied for every subsequence of the zeroes of $b$, completing the proof. 
Now we are ready to prove that (3.a) through (3.f) of Theorem 3 are all equivalent to each other. All the implications are easy to prove except the last one in the chain, namely (3.f) $\Rightarrow(3 . a)$, which requires the use of Theorem 1 .

THEOREM 3.a-3.f. Let $f, g \in H^{\infty}$. Then

$$
(3 . \mathrm{a}) \Leftrightarrow(3 . \mathrm{b}) \Leftrightarrow(3 . \mathrm{c}) \Leftrightarrow(3 . \mathrm{d}) \Leftrightarrow(3 . \mathrm{e}) \Leftrightarrow(3 . \mathrm{f}) .
$$

ProOF. First suppose that (3.a) holds. Our formula for $\left|\left(f \circ L_{\varphi}\right)^{\prime}(w)\right|$, along with the corresponding formula for $\left|\left(g \circ L_{\varphi}\right)^{\prime}(w)\right|$, shows that

$$
\left(f \circ L_{\varphi}\right)^{\prime}(w)\left(g \circ L_{\varphi}\right)^{\prime}(w)=0 \quad \text { for every } w \in D,
$$

which implies that either $f \circ L_{\varphi}$ or $g \circ L_{\varphi}$ is constant on $D$. Because the range of $L_{\varphi}$ is $P(\varphi)$, we conclude that either $f$ or $g$ is constant on $P(\varphi)$, completing the proof that $(3 . a) \Rightarrow(3 . b)$.

Now suppose that (3.b) holds. Let $u \in H^{\infty}[\bar{f}] \cap H^{\infty}[\bar{g}]$. Let $\varphi \in M \backslash D$. To prove that (3.c) holds we must show that $u \circ L_{\varphi} \in H^{\infty}$. By (3.b), either $f \circ L_{\varphi}$ or $g \circ L_{\varphi}$ is constant. Without loss of generality, assume that $f \circ L_{\varphi}$ is constant. Thus if $h_{0}, \ldots, h_{n} \in H^{\infty}$, then

$$
\left(h_{0}+h_{1} \bar{f}+h_{2} \bar{f}^{2}+\cdots+h_{n} \bar{f}^{n}\right) \circ L_{\varphi} \in H^{\infty} .
$$

However, $u \circ L_{\varphi}$ can be uniformly approximated on $D$ by functions of the above form, so $u \circ L_{\varphi} \in H^{\infty}$, completing the proof that (3.b) $\Rightarrow(3 . c)$.

Now suppose that (3.c) holds. Let $h$ be a function in $H^{\infty}$ such that $\bar{h} \in H^{\infty}[\bar{f}] \cap$ $H^{\infty}[\bar{g}]$. Let $\varphi \in M \backslash D$. By (3.c), $\bar{h} \circ L_{\varphi} \in H^{\infty}$. The complex conjugate of this function, namely $h \circ L_{\varphi}$, is also in $H^{\infty}$. Thus $h \circ L_{\varphi}$ is constant on $D$, which means that $h$ is constant on $P(\varphi)$, which implies [11, Chapter X, Exercise 11.b] that $h$ is in the little Bloch space, completing the proof that (3.c) $\Rightarrow(3 . \mathrm{d})$.

Now suppose that (3.d) holds. Let $b$ be an interpolating Blaschke product with zeroes $\left\{w_{n}\right\}_{n=1}^{\infty}$. Simple calculus shows that

$$
\left(1-\left|w_{n}\right|^{2}\right)\left|b^{\prime}\left(w_{n}\right)\right|=\prod_{\substack{m=1 \\ m \neq n}}^{\infty} \rho\left(w_{m}, w_{n}\right) .
$$

Because $b$ is interpolating, the above equation shows that $b$ is not in the little Bloch space. Because (3.d) holds, we can conclude that the complex conjugate of $b$ is not in $H^{\infty}[\bar{f}] \cap H^{\infty}[\bar{g}]$, completing the proof that (3.d) $\Rightarrow(3 . \mathrm{e})$.

Obviously (3.e) $\Rightarrow(3 . f)$.

Finally, suppose that (3.f) holds. If (3.a) does not hold, then there is a thin sequence $\left\{w_{n}\right\}_{n=1}^{\infty}$ such that

$$
\liminf _{n \rightarrow \infty}\left(1-\left|w_{n}\right|^{2}\right)\left|f^{\prime}\left(w_{n}\right)\right|>0 \text { and } \liminf _{n \rightarrow \infty}\left(1-\left|w_{n}\right|^{2}\right)\left|g^{\prime}\left(w_{n}\right)\right|>0 .
$$

Let $b$ be the thin Blaschke product associated with $\left\{w_{n}\right\}_{n=1}^{\infty}$. By Theorem 1, the complex conjugate of $b$ is in $H^{\infty}[\bar{f}] \cap H^{\infty}[\bar{g}]$, contradicting (3.f), so the proof is finished.

5. Doubly commuting multiplication operators. In this section we prove the part of Theorem 3, namely (3.g) $\Rightarrow(3 . \mathrm{a})$, that motivated the earlier parts of the paper. A pair of elements in a $C^{*}$-algebra is called doubly commuting if each 
element in the pair commutes with the adjoint of the other element. Thus (3.g) could be rephrased to state that

$\left\{T_{f}+K\left(L_{a}^{2}\right), T_{g}+K\left(L_{a}^{2}\right)\right\}$ is doubly commuting in the Calkin algebra $B\left(L_{a}^{2}\right) / K\left(L_{a}^{2}\right)$;

here $B\left(L_{a}^{2}\right)$ denotes the bounded operators on $L_{a}^{2}$ and $K\left(L_{a}^{2}\right)$ denotes the compact operators on $L_{a}^{2}$. Before considering the question of when two multiplication operators on the Bergman space doubly commute in the Calkin algebra, one should ask when two multiplication operators doubly commute in $B\left(L_{a}^{2}\right)$. We will answer this question before proving that (3.g) implies (3.a)

To determine when two multiplication operators on the Bergman space are doubly commuting, we need to consider Toeplitz operators. Let $Q$ denote the orthogonal projection of $L^{2}(D, d A)$ onto $L_{a}^{2}$. For $f \in L^{\infty}(D, d A)$, the Toeplitz operator with symbol $f$, denoted $T_{f}$, is the operator from $L_{a}^{2}$ to $L_{a}^{2}$ defined by $T_{f} h=Q(f h)$. Note that if $f \in H^{\infty}$, then this definition agrees with our previous notation for multiplication operators.

It is easy to check that if $f, g \in L^{\infty}$ and $h \in H^{\infty}$, then

$$
\begin{gathered}
T_{f}+T_{g}=T_{f+g} ; \quad T_{g}^{*}=T_{\bar{g}} \\
T_{g h}=T_{g} T_{h} ; \quad T_{\bar{h} g}=T_{\bar{h}} T_{g} .
\end{gathered}
$$

On the Hardy space of the circle, one can determine when two multiplication operators are doubly commuting by representing the operators as matrices with respect to the usual orthonormal basis and then using simple matrix manipulations (see [4, Theorem 9]). We have been unable to make this approach work on the Bergman space, where the matrix representation (with respect to the usual orthonormal basis) of a multiplication operator does not have the nice form (constant on diagonals) associated with a Hardy space Toeplitz operator.

The proof that two multiplication operators on the Bergman space doubly commute only if one of them is a constant multiple of the identity will use the following theorem, which was proved by Sheldon Axler and Allen Shields [3, Theorem 1]. Actually, we only need the following result in the case when $u$ is the complex conjugate of an analytic function; the proof given in [3] can be shortened in this case. However, we state the theorem in the more general form because in the final section of the paper we want to discuss some questions suggested by this theorem and Theorems 1, 2, and 3 .

THEOREM 6. Let $u$ be a bounded, complex-valued, harmonic function on $D$. If $u$ is not analytic on $D$, then $\bar{z} \in H^{\infty}[u]$.

Now we can prove that a multiplication operator on the Bergman space commutes with the adjoint of another multiplication operator only in the trivial case.

THEOREM 7. Let $f, g \in H^{\infty}$. Then $T_{f} T_{g}^{*}=T_{g}^{*} T_{f}$ if and only if either $f$ or $g$ is constant.

PROOF. One direction is trivial; if either $f$ or $g$ is constant, then clearly $T_{f} T_{g}^{*}=$ $T_{g}^{*} T_{f}$.

To prove the other direction, suppose that $T_{f} T_{g}^{*}=T_{g}^{*} T_{f}$, which means that $T_{f}$ commutes with $T_{\bar{g}}$. If $n$ is a nonnegative integer, then $T_{\bar{g}^{n}}=\left(T_{\bar{g}}\right)^{n}$, and so $T_{f}$ 
commutes with $T_{\bar{g}^{n}}$. If $h \in H^{\infty}$, then

$$
T_{f} T_{\bar{g}^{n} h}=T_{f} T_{\bar{g}^{n}} T_{h}=T_{\bar{g}^{n}} T_{f} T_{h}=T_{\bar{g}^{n}} T_{h} T_{f}=T_{\bar{g}^{n} h} T_{f}
$$

Thus $T_{f}$ commutes with $T_{q}$ for every $q$ in $H^{\infty}[\bar{g}]$. If $g$ is not constant, then by Theorem $6 T_{f}$ commutes with $T_{\bar{z}}$. Taking adjoints, we conclude that $T_{z}$ commutes with $T_{\bar{f}}$. However, every operator that commutes with $T_{z}$ is multiplication by some $H^{\infty}$ function [9, Chapter III, Theorem 10.28]. Thus there is a function $h \in H^{\infty}$ such that $T_{\bar{f}}=T_{h}$. If $m$ and $n$ are nonnegative integers, then

$$
0=\left\langle T_{\bar{f}-h} z^{m}, z^{n}\right\rangle=\int_{D}(\bar{f}-h) z^{m \overline{z^{n}}} d A .
$$

By the Stone-Weierstrass Theorem, the linear span of

$$
\left\{z^{m} \overline{z^{n}}: m \text { and } n \text { are nonnegative integers }\right\}
$$

is dense in $C(\bar{D})$. Thus $\bar{f}=h$, which implies that $\bar{f}$ is in $H^{\infty}$, which implies that $f$ is constant, completing the proof.

We are now ready to prove the main result in this section, which states that condition (3.g) of Theorem 3 implies (3.a). Note that the proof of this part of Theorem 3 uses a part of Theorem 3 that was proved earlier in the paper.

THEOREM 3.g. Let $f, g \in H^{\infty}$. If $T_{f} T_{g}^{*}-T_{g}^{*} T_{f}$ is compact, then

$$
\left(1-|z|^{2}\right) \min \left\{\left|f^{\prime}(z)\right|,\left|g^{\prime}(z)\right|\right\} \rightarrow 0 \quad \text { as }|z| \uparrow 1 .
$$

ProOF. Assume that $f, g \in H^{\infty}$ and that $T_{f} T_{g}^{*}-T_{g}^{*} T_{f}$ is compact. Let $h \in H^{\infty}$ be such that $\bar{h}$ is contained in $H^{\infty}[\bar{f}] \cap H^{\infty}[\bar{g}]$. We have already proved that (3.a) is equivalent to (3.d), so to complete the proof we need only show that $h$ is in the little Bloch space.

Our hypothesis states that $T_{f}+K\left(L_{a}^{2}\right)$ commutes with $T_{\bar{g}}+K\left(L_{a}^{2}\right)$ in the Calkin algebra $B\left(L_{a}^{2}\right) / K\left(L_{a}^{2}\right)$. Thus if $n$ is a nonnegative integer, then $T_{f}+K\left(L_{a}^{2}\right)$ commutes with $T_{\bar{g}^{n}}+K\left(L_{a}^{2}\right)$, and so $T_{f} T_{\bar{g}^{n}}-T_{\bar{g}^{n}} T_{f}$ is compact. If $q \in H^{\infty}$, then

$T_{f} T_{\bar{g}^{n} q}-T_{\bar{g}^{n} q} T_{f}=T_{f} T_{\bar{g}^{n}} T_{q}-T_{\bar{g}^{n}} T_{q} T_{f}=T_{f} T_{\bar{g}^{n}} T_{q}-T_{\bar{g}^{n}} T_{f} T_{q}=\left(T_{f} T_{\bar{g}^{n}}-T_{\bar{g}^{n}} T_{f}\right) T_{q}$,

and thus $T_{f} T_{\bar{g}^{n} q}-T_{\bar{g}^{n} q} T_{f}$ is compact. Because $\bar{h} \in H^{\infty}[\bar{g}]$, we can now conclude that $T_{f} T_{\bar{h}}-T_{\bar{h}} T_{f}$ is compact. Taking adjoints, we see that $T_{h} T_{f}^{*}-T_{f}^{*} T_{h}$ is compact.

Repeat the above argument, with $f$ replacing $g$ and $h$ replacing $f$, to conclude that $T_{h} T_{h}^{*}-T_{h}^{*} T_{h}$ is compact. Because (2.g) implies (2.a), we now see that $h$ is in the little Bloch space, completing the proof.

6. Questions and comments. The results in this paper raise many questions, which are discussed in this section.

Let $d \theta$ denote the usual arc length measure on the unit circle $\partial D$, and let $H^{\infty}(\partial D)$ denote the subalgebra of $L^{\infty}(\partial D, d \theta)$ consisting of those functions that are the radial limits almost everywhere $(d \theta)$ of $H^{\infty}$ functions. A rich theory describes the structure of the closed algebras between $H^{\infty}(\partial D)$ and $L^{\infty}(\partial D, d \theta)$. As discussed below, our results seem to hint that a comparable structure may exist for algebras between $H^{\infty}$ and $L^{\infty}(D, d A)$. 
If $u$ is an arbitrary bounded measurable function on $D$, then no one expects the algebra $H^{\infty}[u]$ to have a nice description. However, if $u$ is the complex conjugate of a bounded analytic function, or more generally, if $u$ is a complex-valued bounded harmonic function on $D$, then we suspect that many results about algebras on the circle have analogues for $H^{\infty}[u]$. For example, on the circle, the minimal closed algebra strictly bigger than $H^{\infty}(\partial D)$ is $H^{\infty}(\partial D)+C(\partial D)$. Theorem 6, along with Walter Rudin's theorem (see [17, Theorem 2.13 and 3, Theorem 3]) that $H^{\infty}[\bar{z}]=H^{\infty}+U C(D)$, provides the analogous result for the disk; here $U C(D)$ denotes the set of complex-valued, uniformly continuous functions on $D$.

Let $A O P$ (which stands for "analytic on parts") denote the closed algebra defined by

$$
A O P=\left\{u \in C(M): u \circ L_{\varphi} \in H^{\infty} \text { for every } \varphi \in M \backslash D\right\} .
$$

Because $A O P$ contains the complex conjugate of every Blaschke product in $B_{0}$, we see that $A O P$ is strictly larger than $H^{\infty}+U C(D)$, which does not contain the complex conjugate of any Blaschke product with infinitely many zeroes. Despite the comments in the preceding paragraph, we have seen that $A O P$, rather than $H^{\infty}+$ $U C(D)$, is often the appropriate algebra on the disk to replace $H^{\infty}(\partial D)+C(\partial D)$. For example, on the Hardy space, $H^{\infty}(\partial D)+C(\partial D)$ is the algebra that determines the compactness of certain commutators (and semicommutators) of Hardy space Toeplitz operators (see [2 and 21]). Theorems 2 and 3 show that on the Bergman space, the algebra $A O P$ plays this role.

Is $A O P$ generated, as a closed algebra on $D$, by $H^{\infty}$ and the complex conjugates of the Blaschke products (or inner functions) in the little Bloch space $B_{0}$ ? Let $C O P$ (which stands for "constant on parts") be defined by

$$
C O P=\{u \in C(M): u \text { is constant on } P(\varphi) \text { for every } \varphi \in M \backslash D\} .
$$

(Other authors have defined $C O P$ to be the set of functions in $H^{\infty}$ that are constant on each part in $M \backslash D$; to make the notation on the disk consistent with the notation for $V M O$ and $V M O A$ on the circle, the algebra denoted by others as $C O P$ should be denoted $C O P A$.) Is $A O P=H^{\infty}+C O P$ ? Is $H^{\infty}+C O P$ a closed subalgebra of $C(M)$ ?

The Chang-Marshall Theorem (see [8 and 15]) states that every closed algebra between $H^{\infty}(\partial D)$ and $L^{\infty}(\partial D, d \theta)$ is generated (as a closed algebra) by $H^{\infty}(\partial D)$ and the complex conjugates of some set of interpolating Blaschke products. Theorem 1 suggests that an analogous theorem might be true on the disk. Specifically, if $f \in H^{\infty} \backslash B_{0}$, is $H^{\infty}[\bar{f}]$ generated, as a closed algebra on $D$, by $H^{\infty}$ and the complex conjugates of the interpolating Blaschke products in $H^{\infty}[\bar{f}]$ ? Theorem 1 should be useful in answering this question, since Theorem 1 produces many thin Blaschke products whose complex conjugates are in $H^{\infty}[\bar{f}]$.

Does Theorem 1 remain true if the word "thin" is replaced by "interpolating"? Does Theorem 1 remain true if the word "thin" is replaced by "Blaschke"? If $b$ is an interpolating Blaschke product, is the complex conjugate of every subproduct of $b$ in $H^{\infty}[\bar{b}]$ ? In other words, does Corollary 5 remain true if the word "thin" is deleted? Our guess is that thin Blaschke products play a special role in this theory and that all questions in this paragraph have negative answers. Thin sequences have appeared in other areas of function theory. For example, Carl Sundberg and Thomas Wolff have proved [20, Theorem 1] that the thin sequences are precisely 
the interpolating sequences for the functions of vanishing mean oscillation (and for the bounded analytic functions with vanishing mean oscillation).

Theorem 7 suggests the following question: When do two Toeplitz operators on the Bergman space commute? Two Toeplitz operators on the Hardy space commute if and only if both symbols are analytic or the complex conjugates of both symbols are analytic or one symbol is a linear function of the second symbol (see [4, Theorem 9]). Paul Bourdon has pointed out to us that the corresponding result is false for Bergman space Toeplitz operators. In particular, Bourdon noticed that if $f$ and $g$ are bounded, measurable, radial functions on $D$ (a function is called radial if its value at $z$ depends only on $|z|)$, then $T_{f}$ commutes with $T_{g}$.

G. McDonald and C. Sundberg proved a remarkable theorem that describes the abelianization of the $C^{*}$-algebra generated by all the multiplication operators on the Bergman space. This abelianization (see [16, Theorem 6]) is described in terms of the parts of the maximal ideal space of $H^{\infty}$, as is the condition for two multiplication operators to be doubly commuting in the Calkin algebra [the equivalence of conditions (3.g) and (3.b) of Theorem 3]. What is the connection between these results?

\section{REFERENCES}

1. Sheldon Axler, The Bergman space, the Bloch space, and commutators of multiplication operators, Duke Math. J. 53 (1986), 305-332.

2. Sheldon Axler, Sun-Yung A. Chang, and Donald Sarason, Products of Toeplitz operators, Integral Equations Operator Theory 1 (1978), 285-309.

3. Sheldon Axler and Allen Shields, Algebras generated by analytic and harmonic functions, Indiana Univ. Math. J. 36 (1987), 631-638.

4. Arlen Brown and P. R. Halmos, Algebraic properties of Toeplitz operators, J. Reine Angew. Math. 213 (1964), 89-102.

5. Paul Edward Budde, Support sets and Gleason parts of $M\left(H^{\infty}\right)$, Ph.D. thesis, Univ. of California, Berkeley, 1982.

6. Lennart Carleson, An interpolation problem for bounded analytic functions, Amer. J. Math. 80 (1958), 921-930.

7. Interpolations by bounded analytic functions and the corona problem, Ann. of Math. 76 (1962), 547-559.

8. Sun-Yung A. Chang, A characterization of Douglas subalgebras, Acta Math. 137 (1976), 81-89.

9. John B. Conway, Subnormal operators, Pitman, London, 1981.

10. Theodore W. Gamelin, Uniform algebras, Prentice-Hall, Englewood Cliffs, N. J., 1969.

11. John B. Garnett, Bounded analytic functions, Academic Press, New York, 1981.

12. Håkan Hedenmalm, Thin interpolating sequences and three bounded algebras of bounded functions, Proc. Amer. Math. Soc. 99 (1987), 489-495.

13. Kenneth Hoffman, Bounded analytic functions and Gleason parts, Ann. of Math. 86 (1967), 74-111.

14. Keiji Izuchi, Bloch functions and Hankel operators on Bergman spaces in several variables, preprint.

15. Donald E. Marshall, Subalgebras of $L^{\infty}$ containing $H^{\infty}$, Acta Math. 137 (1976), 91-98.

16. G. McDonald and S. Sundberg, Toeplitz operators on the disc, Indiana Univ. Math. J. 28 (1979), 595-611.

17. Walter Rudin, Spaces of type $H^{\infty}+C$, Ann. Inst. Fourier (Grenoble) 25 (1975), 99-125.

18. Donald Sarason, Blaschke products in $B_{0}$, Linear and Complex Analysis Problem Book, Lecture Notes in Math., vol. 1043, Springer-Verlag, Berlin, 1984.

19. Karel Mattheus Rudolf Stroethoff, Characterizations of the Bloch space and related spaces, Ph.D. thesis, Michigan State Univ., 1987. 
20. Carl Sundberg and Thomas H. Wolff, Interpolating sequences for $Q A_{B}$, Trans. Amer. Math. Soc. 276 (1983), 551-581.

21. A. L. Volberg, Two remarks concerning the theorem of S. Axler, S.-Y. A. Chang and D. Sarason, J. Operator Theory 7 (1982), 209-218.

22. Dechao Zheng, Hankel operators and Toeplitz operators on the Bergman space, preprint.

Department of Mathematics, Michigan State University, East Lansing, MICHIGAN 48824

Department of Mathematics, Bucknell University, Lewisburg, PennsylvaNIA 17837 